\title{
A study on the seroprevalence of cytomegalovirus infection in patients with coronary artery disease in a tertiary care hospital
}

\author{
Prabhu Vairavel ${ }^{1}$, C.P. Ramani ${ }^{2, *}$ \\ ${ }^{1}$ Pre-final M.B.B.S, Madras Medical College, Chennai, Tamil Nadu, ${ }^{2}$ Professor, Institute of Microbiology, Madras Medical \\ College, Chennai, Tamil Nadu, India
}

*Corresponding Author:

Email: cpramani68@gmail.com

\begin{abstract}
Introduction: Coronary artery disease (CAD) is a leading cause of death in developing and developed countries. Infectious etiology is also suspected to be a significant risk factor in these cases. CMV is a beta herpes virus. Once infected, the person carries the virus for life.80\% adults show CMV specific antibodies in their serum indicating the high prevalence of CMV infection.

Aim: To know the seroprevalence of CMV infection in patients with CAD

Materials and Methods: Clinically stable patients undergoing coronary angiography because of symptoms suspected of CAD were included in the study. The samples were tested for specific anti-CMV IgG antibodies by ELISA

Results: Of 150 patients, $86 \%$ had detectable quantities of anti-CMV IgG antibodies in their serum. 15 patients (10\%) were found to be 'borderline positive,' and the antibody index was between 0.9 to 1.1 . 6 patients (4\%) did not have detectable quantities of anti-CMV IgG antibodies in their serum. Prevalence of CAD was $100 \%$ in the study group.

Conclusion: Cytomegalovirus is an essential cause of atherosclerosis due to the infectious burden. In the study population, $86 \%$ of the patients showed elevated levels of anti-CMV IgG antibody. So, there is an association between CMV infection and coronary artery disease.
\end{abstract}

Keywords: Coronary artery diseases, Cytomegalovirus, Infection.

\section{Introduction}

Coronary artery disease $(\mathrm{CAD})$ is a leading cause of death in developing and developed countries. The cause is multifactorial. It is well known that hypertension, hyperlipidemia, obesity, smoking, diabetes and positive family history are the traditional risk factors for atherosclerosis including coronary artery disease. However, these factors are only part of the attributable cardiovascular disease, and some other factors are also involved. ${ }^{1}$

Infectious etiology is also suspected to be an important risk factor in these cases. The association of Chlamydia pneumoniae and coronary artery disease is fairly established. Infection with Helicobacter pylori, cytomegalovirus (CMV) and other herpes viruses are also linked with CAD. But it has not been established. ${ }^{2}$ The role of CMV as a potential factor in the development of cardiovascular disease has been the object of substantial epidemiologic, clinical and laboratory studies.

CMV is a beta herpes virus. Once infected, the person carries the virus for life. $80 \%$ adults show CMV specific antibodies in their serum indicating the high prevalence of CMV infection. ${ }^{3}$

Studies have detected CMV genomic DNA in plasma in coronary atherectomy, myocardial autopsy and donor heart valves and patients with cardiovascular disease. ${ }^{4}$ Heart Outcomes Prevention Evaluation (HOPE) study concludes that the presence of CMV was associated with Myocardial subsequent infarction, stroke and cardiovascular death. ${ }^{5}$
Inflammation plays a central role in coronary artery disease. The chronic inflammatory process including CMV infection has been hypothesized to induce the progression of atherosclerosis in CAD. ${ }^{6}$ The association of markers of chronic infection and CAD has not been adequately evaluated. Hence, this study has been focused on exploring the association of CMV infection and coronary artery disease.

\section{Aim}

1. To know the seroprevalence of CMV infection in patients with CAD.

2. To study the correlation between the anti-CMV antibody titre and CAD.

3. To assess the risk factors associated with CAD.

\section{Materials and Methods}

This cross sectional observational study was conducted in Institute of Microbiology in collaboration with Department of Cardiology in Rajiv Gandhi Government General Hospital (RGGGH), Chennai from July 2017 to August 2017. Clinically stable patients undergoing coronary angiography because of symptoms suspected of CAD such as anginal chest pain, exertional dyspnoea or evidence of myocardial ischemia reported by non-invasive techniques were included in the study.

\section{Exclusion Criteria:}

1. Patients less than 18 years old

2. Patients with acute myocardial infarction 
3. Patients who had previous coronary artery bypass graft or angioplasty

4. Angiogram performed before valvular surgery

Patients with angiographic evidence of atherosclerosis including plaque in any epicardial coronary tree are classified as 'CAD group'.

Those patients with normal angiogram i.e all coronary arteries are judged to be angiographically smooth: 'Non-CAD group'.

History of risk factors such as diabetes, hypertension and smoking were recorded from each patient. Lipid profile and angiographic findings were noted.

Determination of anti-CMV IgG: The samples were tested for specific anti-CMV IgG antibodies by Enzyme Linked Immunosorbent Assay (ELISA).

After calculating the cut-off value from the absorbance of samples and controls, the samples were interpreted as positive or negative as per manufacturer's instructions.

CYTOMEGALOVIRUS (CMV) IgG Elisa: CMV IgG ELISA Kit (Calbiotech) which detects IgG antibody to Cytomegalovirus (CMV) in human serum or plasma.

\section{Results}

Of the one hundred and fifty cases, 81 were male, and 69 were female. 48 patients were between the age group 40-50 years, 33 patients were between 50-55 years, 22 patients were between $55-60$ years, 29 patients were between $60-65$ years and 18 patients were over 65 years old.

All the serum samples used in this study were tested for anti-CMV IgG antibody using Calbiotech ELISA kit.

\section{Fig. 1: Distribution CMV results}

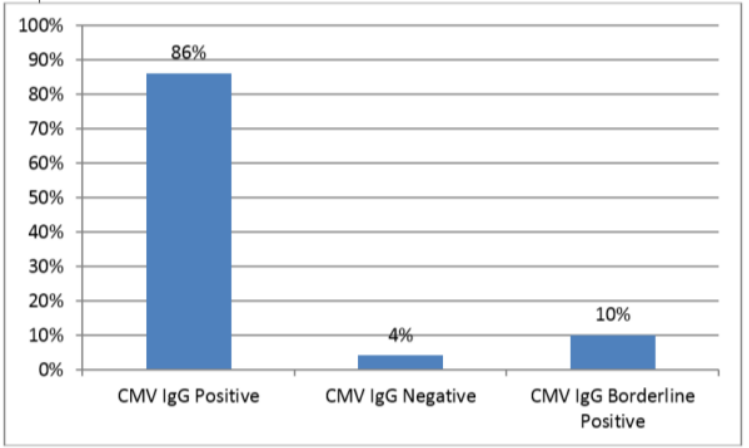

Of 150 patients, 129 patients ( $86 \%$ ) had detectable quantities of anti-CMV IgG antibodies in their serum. These patients exhibited an antibody index of more than 1.1 and were found to be 'seropositive'.

15 patients $(10 \%)$ were found to be 'borderline positive' and the antibody index was between 0.9 to 1.1.

6 patients (4\%) did not have detectable quantities of anti-CMV IgG antibodies in their serum. These patients exhibited an antibody index of less than 0.9 and were found to be 'seronegative'.

All the 150 patients in the study group were diagnosed with coronary artery disease after undergoing coronary angiography.

Hence, the prevalence of CAD was $100 \%$ in CMV seropositive as well as CMV seronegative patients.

The traditional risk factors for CAD and anti-CMV IgG antibody were compared among the patients in the study group.

1. Prevalence of CAD was $100 \%$ in the study group.

2. 42 patients $(28 \%)$ in the study group were diabetics.

3. 69 patients $(46 \%)$ had past history of hypertension.

4. 96 patients $(64 \%)$ had elevated serum lipid levels.

5. 47 patients (31\%) were smokers.
Among the 129 seropositive cases, 36 patients (28\%) had antibody index values between 1.1-1.5. 66 patients $(51 \%)$ had antibody index values between 1.5 2.0. 27 patients $(21 \%)$ had antibody index values more than 2.0.

\section{Discussion}

In recent years, atherosclerosis has come to be recognized as active and inflammatory rather than simply a passive process of lipid infiltration. Inflammation occurs in response to vascular oxidative stress and injury through known and unknown stimuli. ${ }^{7}$

The factors which are responsible for triggering inflammation include oxidized and glycosylated products (e.g., modified lipoproteins). Given their association with inflammation, infectious agents are also being explored as a potential stimulator of vascular inflammation and promoters of atherosclerosis.

Atherosclerosis appears to be of multifactorial etiology. Results of large epidemiological studies have established the concept of traditional risk factors, such as hyperlipidemia, arterial hypertension, diabetes mellitus, smoking and a family history of vascular diseases. ${ }^{1}$ In addition, several studies have reported an 
association between atherosclerosis and certain persistent bacterial and viral pathogens.

Many patients with CAD lack conventional risk factors, suggesting that there are additional unidentified factors contributing to the vascular injury. In regard to this, one of the most interesting developments in recent years has been the idea that infectious agents may induce a pro-inflammatory effect and play a basic role in atherosclerosis. ${ }^{8}$

In our study, out of 150 patients, 129 patients were seropositive for anti-CMV IgG antibody, and 15 patients were in the borderline-positive range. This suggests a strong association of CMV infection with coronary artery disease in our study population.

Upon comparing with traditional risk factors, antiCMV antibody levels were higher in the most number of patients in the study group than hyperlipidemic states, hypertension or diabetes. Hence, CMV infection may be an important risk factor in the pathogenesis of coronary artery disease.

However, other seroepidemiological studies in the past have yielded controversial results. Several authors found an association whereas others did not.

In a study by Dr. Paul D. Sorlie et al. it was found that high levels of CMV antibodies are significantly associated with CAD. The population with the highest antibody levels of CMV IgG showed an increased relative risk (RR) of CAD of 1.76 (95\% CI, 1.8-47.0). After adjusting for age, sex, race and additional covariates of hypertension, diabetes, cigarette smoking, low-density lipoprotein, high-density lipoprotein, and cholesterol levels, RR increased significantly. ${ }^{9}$

In a study by J. M. Ossewaarde, et al it was found that Chlamydia pneumoniae is a risk factor for coronary artery disease in symptom-free elderly men $(\mathrm{OR}=2.76$; $\mathrm{CI}=1.31-5.81)$ and antibodies to CMV or Helicobacter pylori was not associated with CAD risk. ${ }^{8}$

Differences in study design, the frequency of individuals with chronic CMV infection and regional differences may explain the differing results.

However, in our study population, vast numbers of patients have elevated levels of anti-CMV IgG antibodies. It can be explained that these antibodies can contribute to the chronic inflammatory reaction in the vascular endothelium. The chronic inflammation can, in turn, serve as an additional risk factor to traditional risk factors like hyperlipidemia or hypertensive states.

The study by Ji YN, et al stated in a meta-analysis involving 9000 cases, 8608 controls from six prospective studies and 49 retrospective case-control studies. $^{8}$

People exposed to CMV infection had an odds ratio (OR) of $1.67(95 \% \mathrm{CI}, 1.56-1.79)$ for CAD risk, relative to those not exposed.

CMV infection was clearly identified as a risk factor in both prospective studies and retrospective studies.
Interestingly, the increased risk was greater among studies using polymerase chain reaction (PCR) than the risk among studies using ELISA.

Since serologic markers of CMV infection provide tools to follow the natural course of the disease and hitherto, there was no concrete evidence supporting the infection of CMV in the endothelial cells. In our study, serologic markers of CMV infection was measured instead of more sensitive CMV-DNA detection with the hypothesis that circulating CMV-associated antigens might be the risk factors for atherosclerosis. Thus, the link between CMV-DNA positivity and atherosclerosis should be clarified at a larger population in future studies.

\section{Conclusion}

Cytomegalovirus is an important cause of atherosclerosis due to the infectious burden. In the study population, $86 \%$ of the patients showed elevated levels of anti-CMV IgG antibody. So, there is an association between CMV infection and the coronary artery disease in the study population. Chronic CMV infection can promote vascular inflammation, thus can be an important risk factor for atherosclerosis of coronary vessels. In younger patients, screening for CMV will be useful in preventing atherosclerotic heart disease. In such patients, appropriate measures such as administering an anti-viral drug like acyclovir will help lessen the progress of atherosclerosis.

\section{Acknowledgement}

Funded by ICMR Short term Studentship program.

Funding: ICMR.

Conflict of interest: None declared.

\section{References}

1. Nieto FJ. Infections and atherosclerosis: new clues from old hypothesis? Am J Epidemiol 1998;148:937-48.

2. J Danesh, Y Wong, M Waed, J Muir. Chronic infection with Helicobacter pylori, Chlamydia pneumoniae or cytomegalovirus:population based study of coronary artery disease. Circulation 1996;94:922-27.

3. Ananthanarayan, Panikar. Textbook of Microbiology 9th edition; Chapter 51:470-474.

4. Melnick JL, Hu C, Burek J, Adam E, De Bakey ME. Cytomegalovirus DNA in arterial walls of patients with atherosclerosis. J Med Virol 1994;42:170-74.

5. Gkrenia-Klotsas E, Langenberg C, Sharp SJ. Seropositivity and higher immunoglobulin antibody levels against cytomegalovirus are associated with mortality in the European populaton based prospective investigation. Clin Infect Dis 2013;56:1421-27.

6. Ji YN, AN L, Zhan P, Chen XH. Cytomegalovirus infection and coronary artery disease risk:a meta analysis. Mol Biol Rep 2012:6537-46.

7. Corrado E, Novo S. Role of inflammation and infection in vascular disease. Acta Chir Belg 2005;105:567-69.

8. J. M. Ossewaarde, E. J. M. Feskens, C. E. Vallinga. Current infection with Chlamydia pneumoniae, but not seropsitivity to cytomegalovirus, is associated with an 
atherogenic, modified lipid profile. Atherosclerosis, Thrombosis Vascular Biol 2001;21:427-32.

9. Paul D. Sorlie, F. Javier Nieto, Ervin Adam. The Atherosclerosis Risk in Communities (ARIC) Study. Arch Intern Med 2000;160:2027-32.
How to cite this article: Vairavel P., C. Ramani. A study on the seroprevalence of cytomegalovirus infection in patients with coronary artery disease in a tertiary care hospital. Int J Med Microbiol Trop Dis 2018;4(4):27275. 